\title{
Análise bidimensional computadorizada da fenda palpebral
}

\author{
Computerized bidimensional analysis of the palpebral fissure
}

\author{
Antonio Augusto V. Cruz ${ }^{(1)}$ \\ Adriano Baccega ${ }^{(2)}$
}

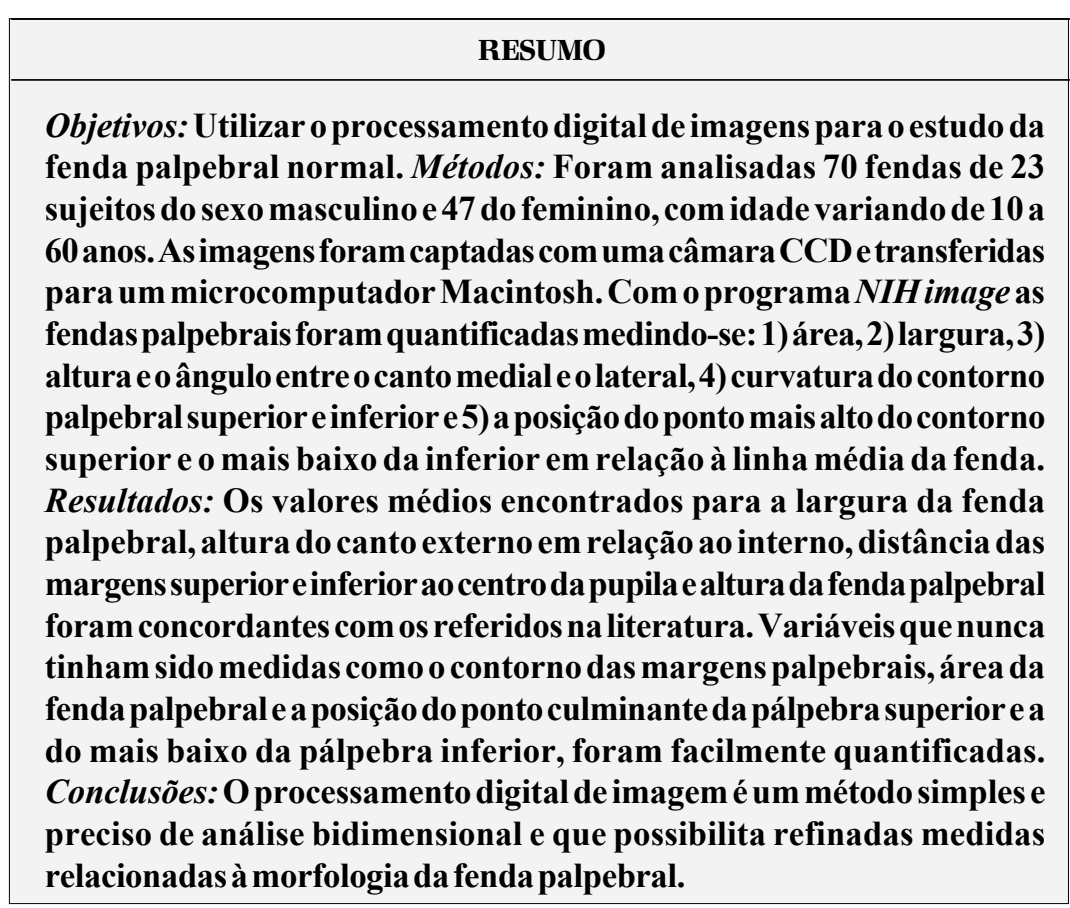

Descritores: Doenças palpebrais/diagnóstico; Pálpebras/anatomia e histologia; Processamento de imagens assistida por computador/métodos.

A fenda palpebral (FP) pode ser definida como o espaço tridimensional delimitado pelas pálpebras superior e inferior. O estudo semiológico da FP normalmente é efetuado quando o indivíduo está em estado de alerta e com os olhos na posição primária do olhar. Nessas condições, a forma da fenda é influenciada por uma complexa interação entre diversos fatores dentre os quais pode-se citar a projeção ântero-posterior do globo ocular, a posição das pálpebras, a altura do canto lateral em relação ao medial, a dimensão horizontal da fenda etc. ${ }^{1}$.

Em certas situações clínicas, a mensuração de determinados elementos constituintes da FP é essencial. Um exemplo típico é o diagnóstico das blefaroptoses e retrações palpebrais que é inteiramente calcado na medida da posição da margem da pálpebra afetada. Além disso, a quantificação da FP é importante em oftalmopediatria, pois conhecimento da evolução pósnatal das dimensões de alguns de seus parâmetros, como a largura ou a distância intercantal interna, é relevante para o diagnóstico de determinadas síndromes ${ }^{2-5}$.

Os dados clínicos relacionados às dimensões da FP em geral são obtidos com instrumentos simples como réguas, trenas ou compassos ${ }^{6}$. Esse tipo de metodologia, que foi extremamente importante para o estabelecimento de valores normativos de variáveis lineares como a distância entre a margem palpebral superior e inferior e o centro pupilar ${ }^{7-9}$, é claramente insuficiente para a medida de outras grandezas mais complexas como curvas, ângulos e áreas. 
Recentemente, técnicas de processamento digital de imagem foram utilizadas, com sucesso, para o estudo do contorno palpebral em diferentes patologias do posicionamento palpebral ${ }^{10}$.

O objetivo desse trabalho é apresentar, pela primeira vez, dados morfométricos da FP palpebral normal obtidos por meio do processamento computadorizado de imagens.

\section{MÉTODOS}

\section{População de estudo}

Foram analisadas as imagens de 70 fendas palpebrais de indivíduos diferentes, sendo 38 imagens do olho direito e 32 do olho esquerdo. Foram tomados os devidos cuidados para se selecionar pessoas que não apresentassem patologias sistêmicas ou oftalmológicas que pudessem alterar a fenda palpebral. A faixa etária variou entre 10 e 60 anos, sendo 23 do sexo masculino e 47 do sexo feminino.

\section{Métodos}

$\mathrm{O}$ registro das imagens da fenda palpebral era efetuado através de uma câmara CCD (Hitachi, KP MIU), ligada a um microcomputador Macintosh (Power-Mac 8100). A captação da imagem era feita a uma distância de 1 metro com a cabeça do sujeito posicionada em uma queixeira. Especial atenção era dada a correção de eventuais inclinações. O sujeito permanecia olhando em frente, para um foco localizado a 6 metros de distância, quando era então captada a imagem.

Para a análise da fenda palpebral foi utilizado o programa NIH 1.55 (escrito por Wayne Rasband no Instituto Nacional de Saúde dos Estados Unidos e encontrado na Internet no endereço: ftp:// zippy.nimh.nih.gov ou disponível em disquetes no endereço NTIS, 5285 Port Royal Rd, Springfield, VA 22161, PB-504868, USA. A técnica básica foi a descrita com detalhes por Cruz e colaboradores ${ }^{10}$.

Como não houve diferença entre os sexos na medida de nenhuma das variáveis que serão descritas abaixo, os dados relativos aos dois sexos foram agrupados para a realização de três experimentos:

\section{Experimento 1}

O contorno da fenda foi delineado com pontos em toda a sua extensão (figura $1 \mathrm{~A}$ ). A partir desse delineamento, mediram-se parâmetros relacionados à fenda como um todo, ou seja: a) área, b) a largura e c) a altura e o ângulo entre o canto medial e o lateral (figura $1 \mathrm{C}$ ).

\section{Experimento 2}

Os contornos palpebrais, delineados com pontos nas margens palpebrais, foram representados graficamente com e sem o segmento lacrimal (figura 2A). Em seguida, comparou-se o ajuste dos pontos dos contornos palpebrais, por meio de funções parabólicas, ou de segundo grau, do tipo $\mathbf{y}=\mathbf{a x ^ { 2 }}+\mathbf{b x}+\mathbf{c}$, tanto para a porção ciliar isoladamente como para toda a margem (porção ciliar + lacrimal) (figura 2B).
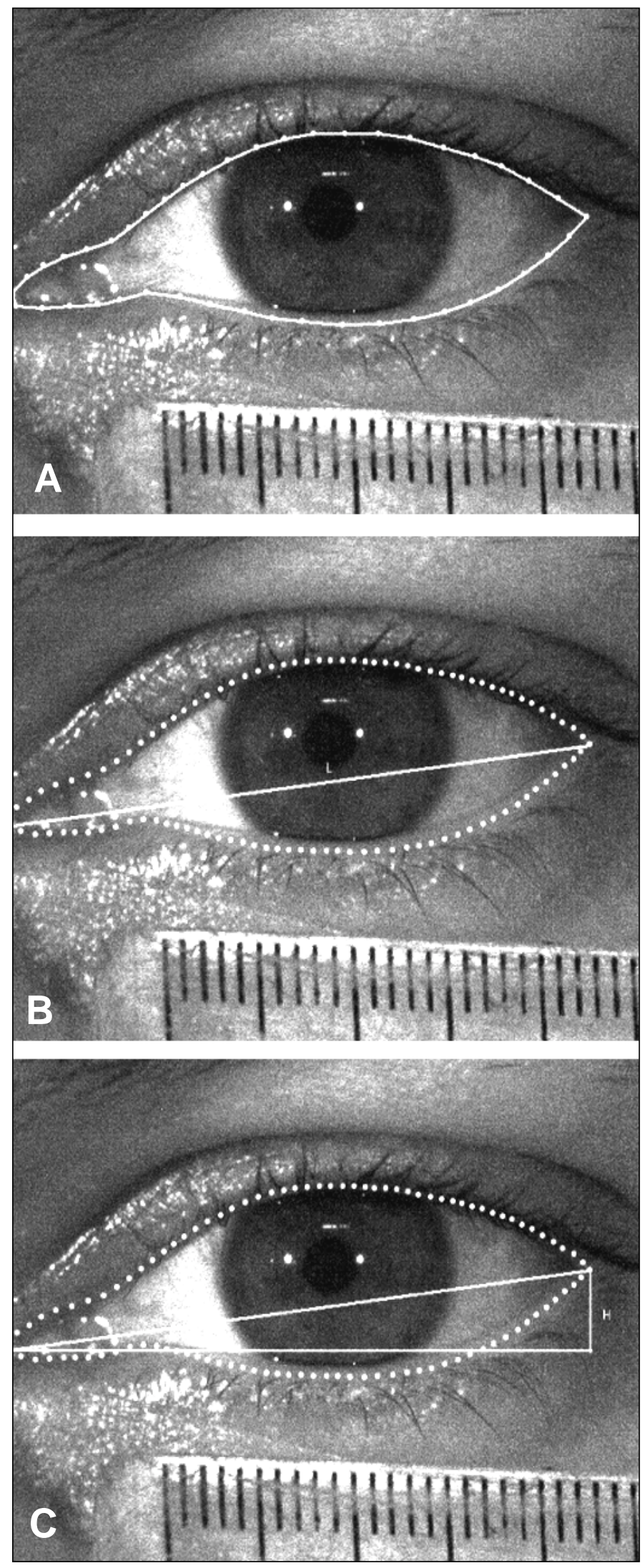

Figura 1 - Digitalização de imagem da fenda palpebral; A) delineamento da área bidimensional da fenda palpebral; B) medida da largura da fenda palpebral; C) medida da altura (H) e ângulo do canto externo. 


\section{Experimento 3}

Após a determinação do melhor ajuste do contorno, mediu-se a curvatura palpebral (parâmetro a da função parabólica), assim como a posição do ponto do contorno da pálpebra superior e o inferior cuja reta tangente tem inclinação igual a zero. Essa medida foi feita determinando-se a posição no eixo das abcissas da derivada da função igual a zero. Como a derivada de uma função do segundo grau do tipo $\mathbf{y}=\mathbf{a x}^{2}+\mathbf{b x}+\mathbf{c}$ é uma linha reta, $\mathbf{d y} / \mathbf{d x}=\mathbf{2} \mathbf{a x}+\mathbf{b}$, o ponto do eixo horizontal corresponde ao pico da curva superior e o mais baixo da inferior, é calculado pela fórmula $\mathbf{2 a x}+\mathbf{b}=\mathbf{0}$ (figura 3A).

As posições desses dois pontos, ou seja, mais alto superior e mais baixo inferior, foram expressas em relação ao ponto médio da fenda palpebral (respectivamente, $\Delta$ sup e $\Delta$ inf); sendo adotado como negativas quando localizados nasalmente ao ponto médio e positivas quando temporais ao mesmo ponto. $\mathrm{O}$ ponto médio da fenda foi tomado como sendo o meio da linha que une os dois cantos (medial e lateral). Mediu-se também a

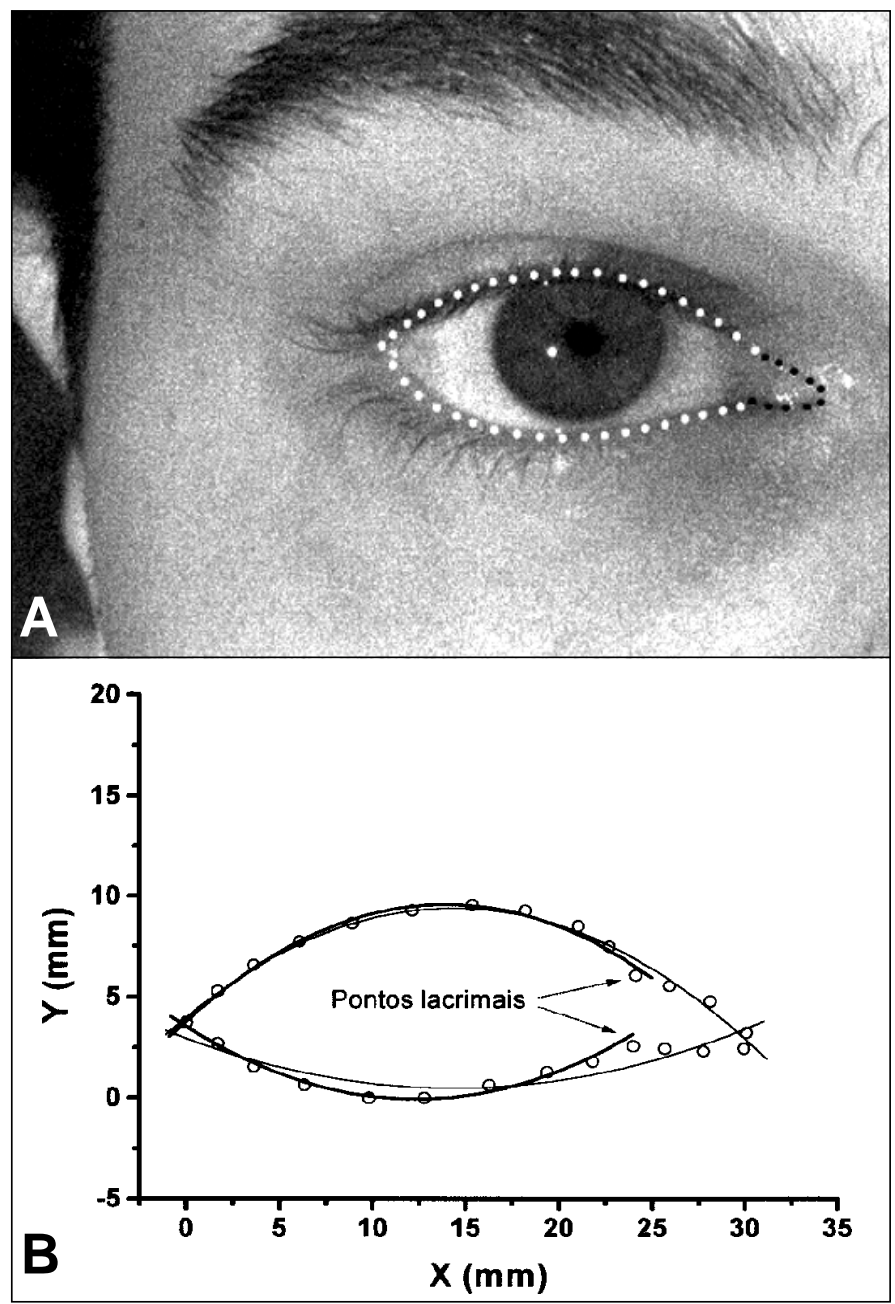

Figura 2 - A) Delineamento das porções ciliar (pontos brancos) e lacrimal (pontos negros) de imagem digitalizada da fenda palpebral; B) Ajuste de funções do segundo grau, para os contornos superior $\mathrm{e}$ inferior,porção ciliar e ciliar + lacrimal.

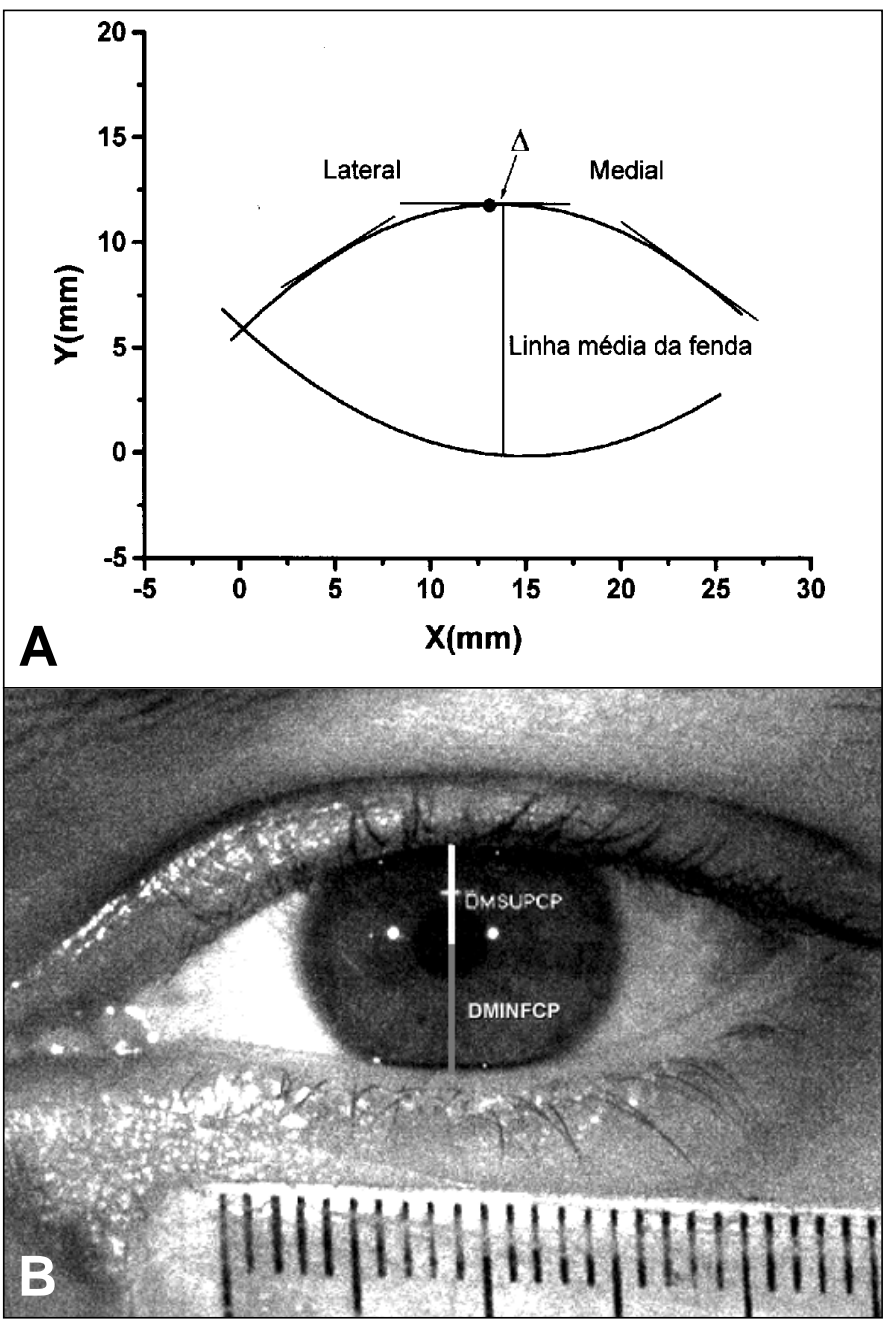

Figura 3 - A) determinação da posição do ponto culminante do contorno superior. A derivada (reta tangente à curva) tem inclinação $=0$. A distância desse ponto à linha média é a variável ); B) medida da distância entre o centro pupilar e a margem superior (DMSUPCP) e inferior (DMINFCP).

distância entre o centro da pupila e a margem palpebral superior (DMSUPCP) e inferior (DMINFCP) (figura 3B).

\section{Análise estatística}

Todas as variáveis tiveram suas distribuições descritas por meio de histogramas e determinação dos clássicos parâmetros média, mediana, desvio padrão, valor máximo e mínimo. Comparações entre mesmas variáveis da pálpebra superior e inferior foram feitas com o teste t de Student para amostras pareadas. Graus de ajustes de funções foram comparados pelo cálculo do coeficiente de determinação $\mathrm{R}^{2}$.

\section{RESULTADOS}

\section{Experimento 1}

A Figura 4 mostra as distribuições da área e largura da 
fenda, altura e ângulo do canto externo em relação ao interno. A tabela 1 apresenta os valores dos principais parâmetros dessas distribuições.

\section{Experimento 2}

A Tabela 2 relaciona os valores médios dos coeficientes de determinação $\mathrm{R}^{2}$ obtidos para os ajustes quadráticos dos contornos palpebrais superior e inferior com e sem a porção lacrimal. Teste t para amostras pareadas mostrou claramente que a exclusão da porção lacrimal melhora sobremaneira o ajuste quadrático dos contornos, especialmente para a pálpebra inferior.

\section{Experimento 3}

$\mathrm{Na}$ Figura 5 estão representadas as distribuições das distâncias entre o centro pupilar e as margens superior (DMSUPCP) e inferior (DMINFCP) e a da altura da fenda, ou seja, a distribuição da soma dos valores DMSUPCP e DMINFCP.

A Figura 6 apresenta as distribuições de parâmetros relacionados aos contornos das pálpebras superior e inferior, ou seja, distribuição da curvatura superior, inferior, posição do ponto culminante superior e mais baixo inferior. A tabela 3 relaciona os principais parâmetros dessas distribuições.
Comparações pareadas com o teste $\mathbf{t}$ indicaram que a curvatura da pálpebra superior é significativamente maior que a da inferior $(\mathbf{t}=7,78, \mathbf{p}<0,00001)$. Já a diferença entre DELTA PSUP e DELTA PINF não foi significativa $(\mathbf{t}=0,86, \mathbf{p}=0,39)$.

\section{DISCUSSÃO}

Digitalizar uma imagem significa atribuir valores espaciais $(\mathrm{x}, \mathrm{y})$ e de luminância aos pontos (pixeis) que compõem a imagem. Uma vez digitalizada, a imagem pode ser processada por programas específicos que, ao manipularem matematicamente os pixeis, permitem o delineamento de bordas, filtragem de determinadas características e, evidentemente, a mensuração de qualquer parâmetro linear ou curvo da imagem ${ }^{11}$. Qualquer microcomputador do tipo PC ou Macintosh pode ser usado para esse tipo de análise, desde que equipado com uma placa capturadora do tipo frame-grabber e uma câmara CCD. Programas específicos para tratamento de imagem são, em geral, fornecidos gratuitamente pelos fabricantes das placas capturadoras.

$\mathrm{O}$ uso do processamento digital de imagem aplicado à morfometria da FP, forneceu um conjunto de dados inteira-

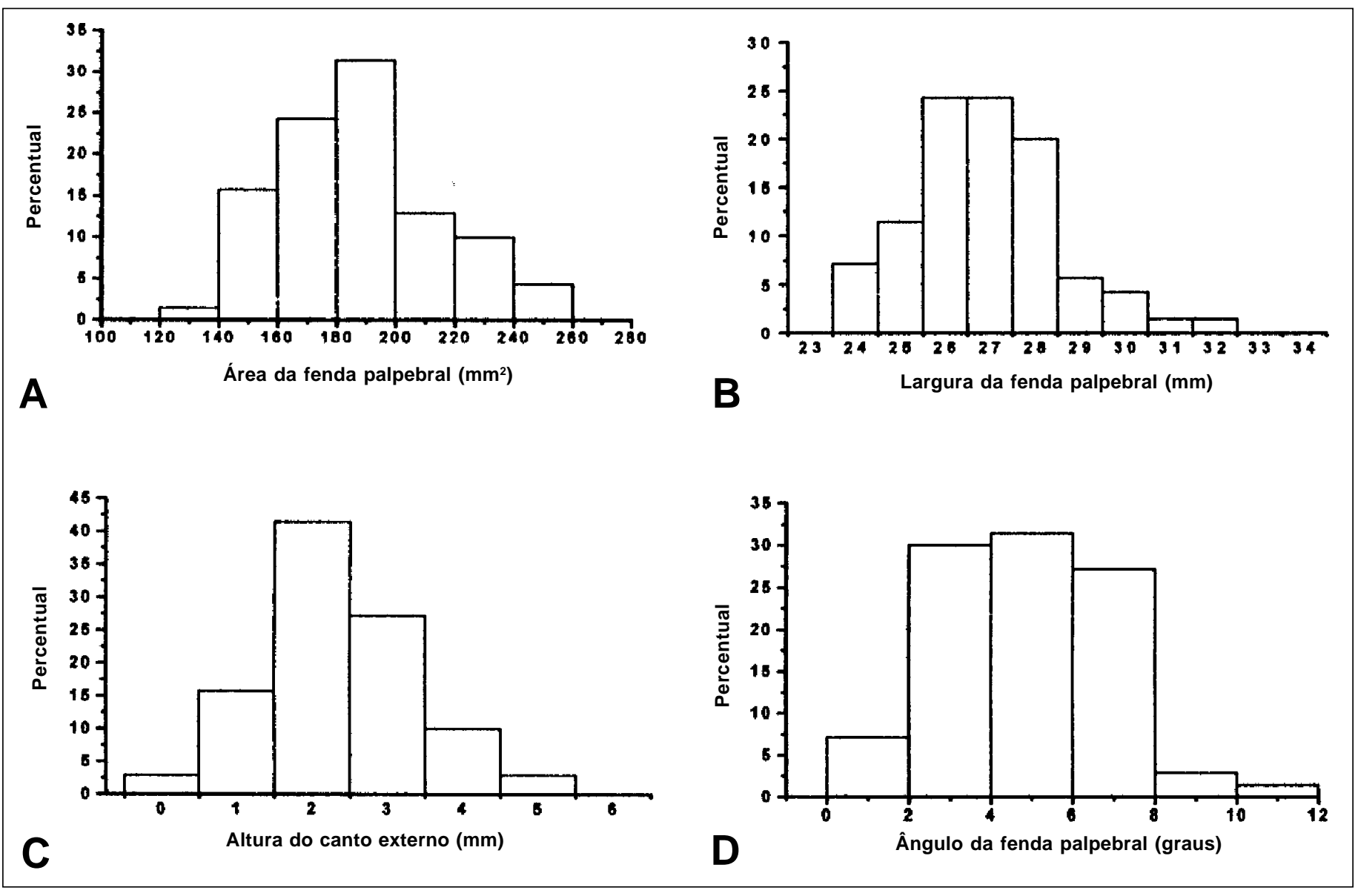

Figura 4 - Distribuições de variáveis ligadas à fenda como um todo: A) área; B) largura; C) altura do canto externo em relação ao interno e D) ângulo da fenda. 

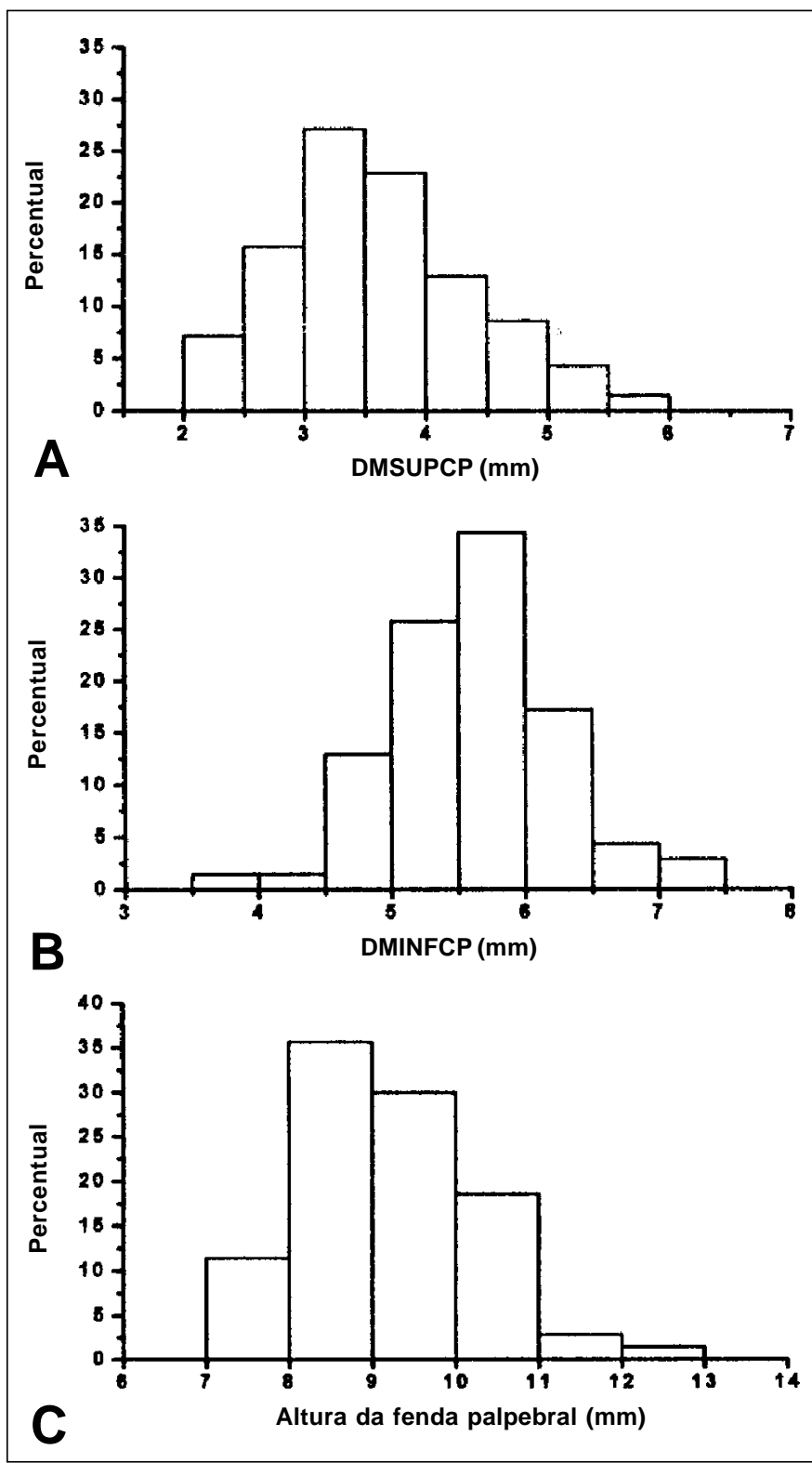

Figura 5 - Distribuições das distâncias entre o centro pupilar e a margem palpebral superior $(A)$ e inferior $(B)$ e da altura da fenda.

mente concordantes com os já citados na literatura e, além disso, possibilitou novas e refinadas análises quantitativas da morfologia da fenda.

Dados concordantes com a literatura foram a largura e a angulação da fenda, bem o posicionamento palpebral. Por exemplo: na literatura, a largura da fenda palpebral de adultos normais é citada como sendo de 28 a $30 \mathrm{~mm}^{12}$. Fox, em seu clássico artigo sobre a fenda palpebral, fornece dados que permitem o cálculo da média da fenda como $28,6 \mathrm{~mm}^{13}$. O valor médio obtido no presente trabalho de $26,82 \mathrm{~mm}$ ( $\mathrm{sd}=0,785)$, é inteiramente concordante com esses valores da literatura. Deve-se lembrar que a quantificação da morfometria da fenda realizada na clínica leva em conta apenas o valor horizontal da fenda palpebral, ou seja, não considera o fato de o canto externo estar, em grande número de sujeitos, acima do interno ${ }^{3,14-15}$. Os valores da altura do ângulo externo e angulação da fenda medidos no presente trabalho (figuras $4 \mathrm{C}$ e $4 \mathrm{D}$ e tabela 1 ) indicam que, ao se obedecer a obliqüidade da fenda, a diferença no cálculo da largura é negligenciável, uma vez que as duas medidas são relacionadas pelo cosseno do ângulo da fenda que é, em média, cerca de 5 graus $\left(\operatorname{cosseno} 5^{\circ}=0,99\right)$. Vale lembrar que a morfometria da fenda é influenciada por características raciais. Assim os dados normativos obtidos no presente trabalho referem-se à uma população geneticamente mista, típica do Brasil. É possível que em outros grupos populacionais, como em orientais, os valores sejam diferentes. O fator idade também não foi estudado no presente trabalho. As modificações induzidas pelo envelhecimento na fenda palpebral serão objeto de um estudo específico.

Outra variável cujos valores normativos da literatura foram confirmados no presente trabalho é a angulação da fenda. A obliqüidade da FP depende, obviamente, da relação entre a altura do canto externo em relação ao interno e a largura da fenda. A altura média encontrada $(2,33 \mathrm{~mm})$ é concordante com os dados da literatura ${ }^{12-13}$. Levando-se em conta as dimensões da largura, a angulação média da fenda foi de 4,82 graus, com valores extremos variando entre 0 e 10,67 graus. $O$ conhecimento da obliqüidade normal da fenda tem importância em oculoplástica, não só do ponto de vista cirúrgico, como também diagnóstico. Angulações negativas (denominadas de antimongóis) são anômalas e, provavelmente, indicam distopia cantal lateral ${ }^{16}$.

Modernamente, o posicionamento palpebral superior e inferior é medido em relação ao reflexo pupilar de uma fonte luminosa fixada na posição primária do olhar. Como a posição do reflexo luminoso é praticamente coincidente com o centro da pupila, as medidas feitas no presente trabalho não foram significativamente diferentes dos valores médios referidos na literatura. Para a distância da margem superior ao centro da pupila (DMSUPCP), o valor médio obtido foi de $3,57 \mathrm{~mm}$, sendo o valor máximo encontrado de 5,53 e o mínimo de 2,08 $\mathrm{mm}$, inteiramente concordante com Frueh, que em 106 sujeitos normais examinados com idade abaixo de 65 anos obteve o valor médio de $3,5 \mathrm{~mm}( \pm 0,9)^{7}$. Para a pálpebra inferior, muitos autores consideram que a posição normal é a tangente ao limbo palpebral inferior ${ }^{17}$. Como o diâmetro vertical da córnea é de aproximadamente $11 \mathrm{~mm}$ e a pupila encontra-se no ponto médio dessa distância, isso equivale dizer que a pálpebra inferior está a aproximadamente $5,5 \mathrm{~mm}$ do centro da pupila. A média obtida da distância da margem inferior ao centro da pupila (DMINFCP) foi de $5,63 \mathrm{~mm}$, ou seja, também concordante com as normas da literatura. Quanto à altura da fenda palpebral (DMSUPCP + DMINFCP), o valor médio obtido foi de 9,02 mm estando de acordo com o descrito por Fox, onde em uma análise de 1686 sujeitos, de 10 a 60 anos, obteve uma média de $9,24 \mathrm{~mm} \cdot{ }^{13}$.

Não conseguimos achar na literatura valores normativos 


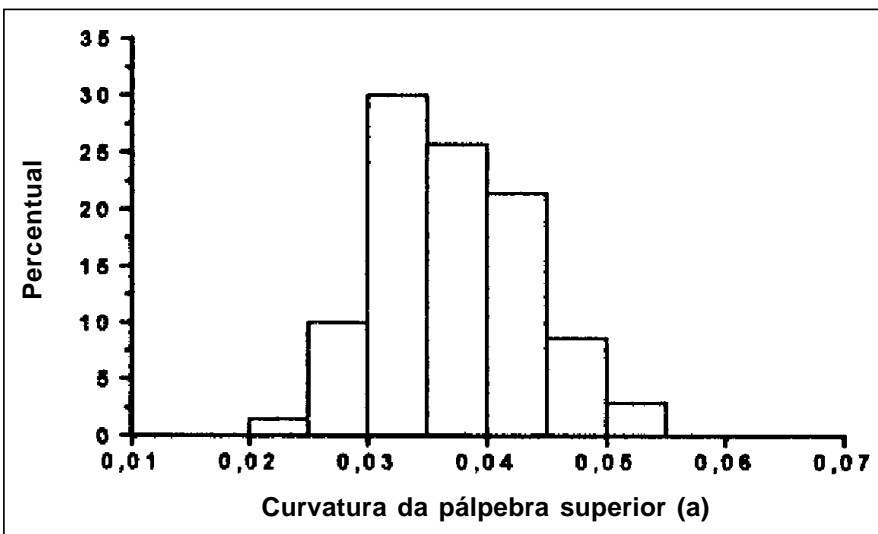

A

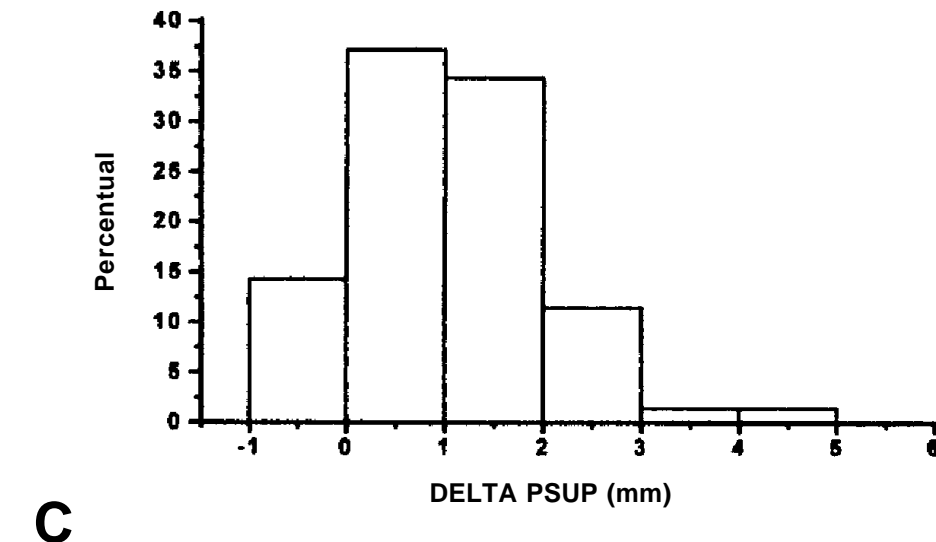

Figura 6 - Distribuições das curvaturas palpebrais superior (A) e inferior (B) e das distâncias entre o ponto culminante superior (C) e inferior(D) em relação a linha média. Notar que deslocamentos temporais foram expressos positivamente e os nasais negativamente.

da área da FP. No presente trabalho, o valor médio obtido foi de $185,98 \mathrm{~mm}^{2}$. Embora a área da fenda nunca tenha sido estudada em diferentes condições patológicas, os sintomas de exposição sofridos por pacientes com proptose e/ou retração palpebral são, provavelmente, melhor correlacionados ao parâmetro área do que a simples medida isolada da exoftalmometria ou posição palpebral. É importante esclarecer que os valores de área medidos a partir de imagens frontais da FP, representam, na verdade, uma aproximação bidimensional de um espaço tridimensional. A área real da FP é um pouco maior principalmente devido a projeção anterior sofrida pelas pálpebras pela presença do olho, como também pelo fato do canto externo estar discretamente posicionado atrás do interno.

Em relação ao problema do ajuste de funções matemáticas para a quantificação do contorno palpebral, a tabela 2 indica que o coeficiente de determinação $\mathrm{R}^{2}$ (parâmetro que mede o grau de ajuste, podendo variar de 1 , ajuste perfeito, a 0 , ausência de ajuste) das funções quadráticas, ajustadas tanto para a pálpebra superior como para inferior, ocorreu mais adequadamente quando se excluiu a porção lacrimal da fenda
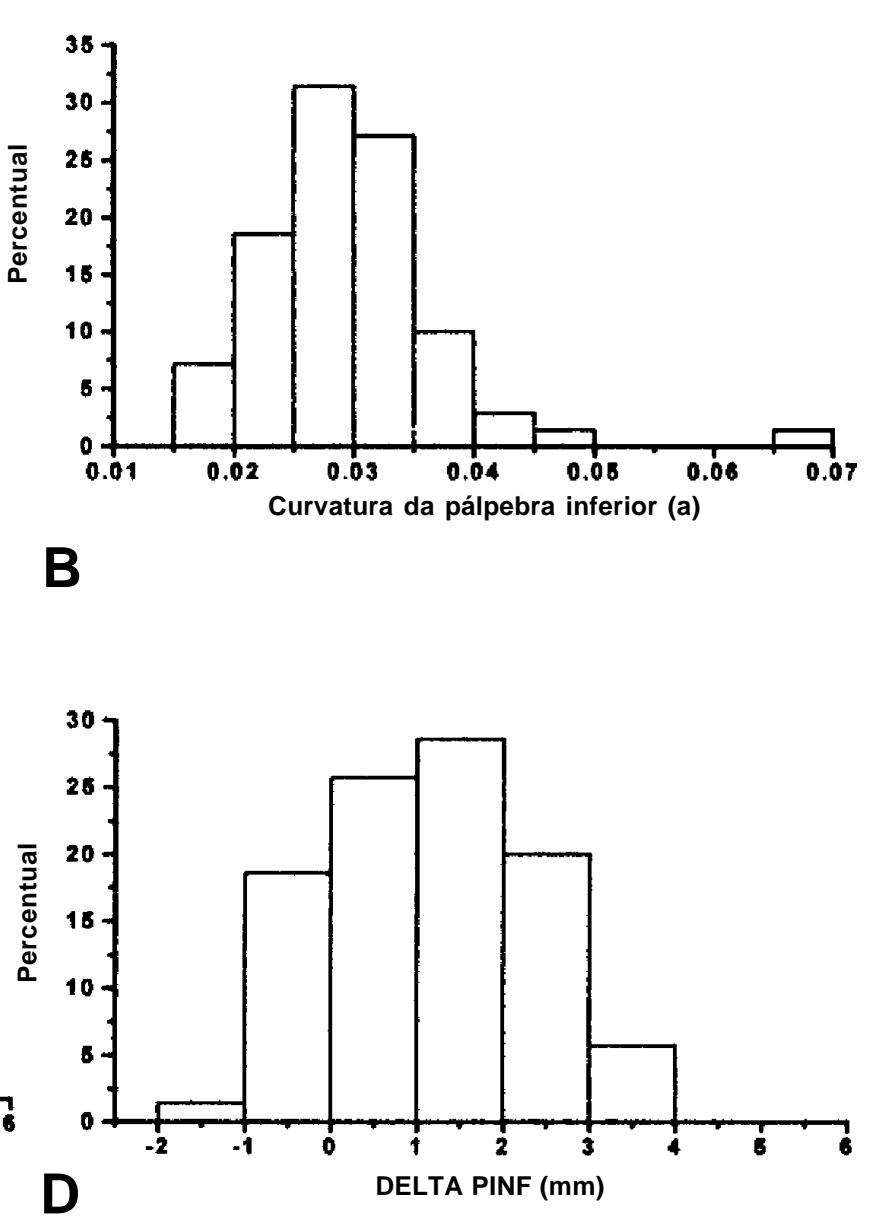

B

palpebral (principalmente para a pálpebra inferior). Esse fato pode ser explicado pela configuração tridimensional da fenda. Como o canto interno ocupa uma posição afastada do globo ocular, o segmento lacrimal das pálpebras não está apoiado no globo. Como apenas o contorno ciliar repousa, de fato, sobre uma superfície esférica, somente essa porção é bem ajustada por curvas do segundo grau. A razão matemática dessa aproximação foi recentemente esclarecida ao se mostrar que um segmento de arco de círculo quando projetado bidimensionalmente pode ser aproximado, na sua porção central, a uma parábola ${ }^{18}$.

O contorno que as margens palpebrais delineiam depende de uma complexa interação de forças ativas (tono muscular dos retratores superior e inferior), e passivas (propriedade elástica dos tecidos), assim como da própria presença do globo ocular. Como a pálpebra inferior apenas tangencia o globo, enquanto que a superior sobre este repousa, a curvatura da superior é significativamente maior que a da inferior $(\mathbf{t}=7,78, \mathbf{p}<0,00001)$.

Os dados do presente trabalho permitiram ainda esclarecer 
alguns detalhes morfológicos que eram descritos de maneira imprecisa na literatura. Por exemplo, existem referências que trazem o ponto culminante da pálpebra superior como localizado medialmente ao centro da pupila, e o mais baixo da pálpebra inferior como situado lateralmente ${ }^{12-13,19}$. Contudo, não há dados quantitativos que demonstrem essa afirmativa $\mathrm{e}$ no único texto em que foi encontrada uma representação pictórica da posição do ponto mais alto superior, ele era localizado como tangente ao limbo medial ${ }^{19}$, o que não condiz com os dados verificados no presente trabalho.

Optamos por analisar o pontos específicos do contorno palpebral em relação à linha média da fenda palpebral e não com respeito ao centro pupilar, em virtude da dificuldade teórica em definir-se a posição primária do olhar. Também, durante cirurgias com anestesia geral e mesmo local, a posição do olho é muito variável, o que torna a utilização do referencial pupilar difícil ou mesmo impossível. De acordo com a tabela 3, para a pálpebra superior o ponto mais alto de sua curvatura encontra-se em média 1,04 mm lateral à linha média da FP e na pálpebra inferior, o mais baixo está $1,19 \mathrm{~mm}$ lateralmente a essa linha. Esses valores são diferentes quando se estuda as pálpebras de sujeitos com retração palpebral por orbitopatia de Graves e blefaroptose ${ }^{10} \mathrm{e}$ têm óbvia implicação cirúrgica na correção dessas patologias ${ }^{20}$.

Em resumo, a aplicação do processamento digital de imagem mostrou-se extremamente profícua para a análise da morfometria da fenda palpebral. Variáveis que nunca tinham sido medidas como área e contorno palpebral foram quantificadas e outros dados lineares como a distância entre a margem palpebral e a pupila, foram validados. No conjunto, os resultados fornecem um referencial para a análise da fenda em diferentes patologias do posicionamento palpebral ou mesmo ocular.

\section{CONCLUSÕES}

O processamento computadorizado de imagens de fenda palpebral possibilita refinadas análises quantitativas de parâmetros de importância clínica e cirúrgica. Variáveis como curvatura palpebral, área e angulação da fenda são facilmente mensuráveis em imagens digitais. A conjugação de fotografias digitais e uso do microcomputador deve fazer parte do arsenal semiótico do cirurgião oculoplástico moderno.

\section{ABSTRACT}

Purpose: To use digital image processing analysis techniques as a clinical tool for measuring the normal palpebral fissure. Methods: 70 palpebral fissure images of 23 males and 47 females with ages ranging from 10 to 60 years were analyzed. The images were captured with a CCD camera and transferred to a Macintosh computer. Using the NIH software, the following variables were measured: 1) area, 2) length, 3) height and angle between the medial and lateral canthi, 4) upper and lower eyelid curvatures 5) position of the highest point of the upper contour and the lowest point of the lower contour relative to the palpebral fissure midline. Results: The mean values obtained for the length, height and vertical position of the lateral canthus relative to medial canthus agree well with normative values found in the literature. Variables that have never been quantified as the upper and lower eyelid contour and palpebral fissure area were easily measured. Conclusions: The digital image processing is a simple method for the 2dimensional analysis of the palpebral fissure and allows refined measurements of the fissure morphology.

Keywords: Eyelid diseases/diagnosis; Eyelids/anatomy \& histology; Image processing, computer-assisted.

\section{REFERÊNCIAS}

1. Lemke BN. Anatomic considerations in upper eyelid retraction. Ophthal Plast Reconstr Surg 1991;7:158-66.

2. Leung AK, Ma KC, Siu TO, Robson WL. Palpebral fissure length in chinese newborn infants. Comparison with other ethnic groups. Clin Pediatr (Phila) 1990;29:172-4

3. Fuchs M, Iosub S, Bingol N, Gromisch DS. Palpebral fissure size revisited. J Pediatr 1980;96:77-8.

4. Thomas IT, Gaitantzis YA, Frias JL. Palpebral fissure lenght from 29 weeks gestation to 14 years. J Pediatr 1987;111:267-8.

5. Jones KL, Hanson JW, Smith DW. Palpebral fissure size in newborn infants. J Pediatr 1978;92:787.

6. Soares EJC, Figueiredo ARP, Souza GL, Almeida HC, Oliveira LRM, Magalhães MM, et al. Blefaroptose. In: Soares EJC, Moura EM, Gonçalves JOR, editors. Cirurgia plástica ocular. São Paulo: Rocca; 1997. p. 77-152.

7. Frueh BR. Graves' eye disease: orbital compliance and other physical measurements. Trans Am Ophthalmol Soc 1984;82:492-598.

8. Small RG. Upper eyelid retraction in Graves'ophthalmopathy: A new surgical technique and a study of the abnormal levator mucle. Trans Am Ophthalmol Soc 1988;86:725-93.

9. Small RG, Sabates NR, Burrows D. The measurement and definition of ptosis. Ophthalmic Plast Reconstr Surg 1989;5:171-5.

10. Cruz AA, Coelho RP, Baccega A, Lucchezi MC, Souza AD, Ruiz EE. Digital image processing measurement of the upper eyelid contour in graves disease and congenital blepharoptosis. Ophthalmology 1998;105:913-8.

11. Souza AD, Ruiz EE, Cruz AA. Palpebral fissure morphology segmentation and measurement using image processing. IEEE Eng Med Biol Mag 2000;19:114-9

12. Bron AJ, Tripathi RC, Tripathi BJ. The ocular appendages: eyelids, conjunctiva and lacrimal apparatus. In: Bron AJ, Tripathi RC, Tripathi BJ, editors. Wolff's anatomy of the eye and orbit. 8th ed. London: Chapman \& Hall Medical; 1997. p. 30-84.

13. Fox SA. The palpebral fissure. Am J Ophthalmol 1966;62:73-8.

14. Iosub S, Fuchs M, Bingol N, Stone RK, Gromisch DS, Wasserman E. Palpebral fissure length in black and Hyspanic children: correlation with head circumference. Pediatrics 1985;75:318-20.

15. Van den Bosch WA, Leenders I, Mulder P. Topographic anatomy of the eyelids, and the effects of sex and age. Br J Ophthalmol 1999;83:347-52.

16. Duke-Elder S. Developmental anomalies of the eyelids. In: Duke-Elder S, editor. Text-book of ophthalmoloy. London: Henry Kimpton; 1952. p. 463293.

17. Shore JW. Changes in lower eyelid resting position, movement, and tone with age. Am J Ophthalmol 1985;99:415-23.

18. Cruz AAV, Baccega A, Oliveira MVD, Malbouisson JM. A geometric model of eyelid contour. Invest Ophthalmol Vis Sci 1999; 40 (Suppl):29.

19. Whitnall SE. Eyelids. In: The anatomy of the human orbit and accessory organs of vision. 2nd ed. v. 1. London: Oxford University Press; 1932. p. 109-252.

20. Cruz AAV, Lucchezi MC. Quantification of palpebral fissure shape in severe congenital blepharoptosis. Ophthal Plast Reconstr Surg 1999;15:232-5. 